\title{
Watch and Wait in Lebanese Chronic Lymphocytic Leukemia Patients: How Relevant is it?
}

\author{
Antoine Abi Lutfallah, Hampig Raphael Kourie*, Roland Eid, Fadi Farhat, \\ Marwan Ghosn, Joseph Kattan
}

\begin{abstract}
Background: Chronic lymphocytic leukemia (CLL) is the most frequent form of leukemia in Europe and North America, and it mainly affects older individuals. Many approaches are implemented in the management of CLL from a watch and wait $(W \& W)$ strategy to chemotherapeutic regimens. We here reviewed our clinical practice for the relevance of the $W \& W$ strategy in Lebanese CLL patients. Materials and Methods: A total of 95 patients with CLL diagnosed in four institutions in Lebanon, between 1992 and 2013, were selected and their files were reviewed. Characteristics of these patients were noted including age, sex, RAI and Binet scores, CBC values, presence of hepatomegaly or splenomegaly, performance of bone marrow biopsy or peripheral blood flux cytometry for diagnosis, adoption of $\mathrm{W} \& \mathrm{~W}$ strategy, different chemotherapeutic regimens and the indications for treatment. Results: Some 38 patients $(40 \%)$ diagnosed with CLL were women and $57(60 \%)$ were men with a mean age of 65.1 years [36-89]. Of the total, $50.5 \%, 17.2 \%, 14 \%, 7.5 \%$ and $10.8 \%$ had an RAI score at diagnosis of $0,1,2,3$ and 4, respectively, while $65.6 \%, 17.2 \%$ and $17.2 \%$ had Binet scores of I, II and III. The mean lymphocyte count at diagnosis was $39885 / \mathrm{mm}^{3}[1596-290000]$, the mean hemoglobin level was $12.7 \mathrm{~g} / \mathrm{dl}$ [6.2-17] and the mean platelet count was $191255 / \mathrm{mm}^{3}[14000-458000]$. While $26.3 \%$ of patients with CLL had splenomegaly, only $7.4 \%$ had hepatomegaly. Some $33.7 \%$ had undergone a bone marrow biopsy, 66.3\% flow cytometry of circulating blood and $5.3 \%$ a lymph node biopsy. Overall, the W\&W was adopted in 62.4\% (58) of patients with a mean duration of 37.7 months [3-216]. The W\&W was used in 82.6\%, 73.3\%, 46.2\%, 14.3\% and $0 \%$ of patients having RAI scores of $0,1,2,3$ and 4 , respectively, and, it was used in $80 \%, 46.7 \%$ and $6.25 \%$ with Binet scores of I, II and III. The most frequent indication for treatment was anemia and thrombocytopenia, accounting for $32.7 \%$ of cases. The most frequently used chemotherapeutical regimens were chlorambucil until the end of the last century and flufarabine-cyclophosphamide-rituximab during the last decade. Conclusions: This retrospective review of CLL clinical practice showed an important implementation of the W\&W strategy with a long duration, especially in early stage cases with low RAI or Binet scores.
\end{abstract}

Keywords: Watch and wait strategy- chronic lymphocytic leukemia - treatment - Lebanon

Asian Pac J Cancer Prev, 17 (1), 215-217

\section{Introduction}

Chronic lymphocytic leukemia (CLL) is the most frequent leukemia in developed countries (Siegel et al., 2015). It occurs more frequently in elderly and in men with a sex ratio of 2/1 (Smith et al., 2011 and Hernandez et al 1995). The diagnosis of CLL is suspected following the detection of lymphocytosis exceeding $5000 / \mu \mathrm{L}$ on a complete blood count and confirmed by flow cytometry immunophenotyping on peripheral blood (Hallek et al., 2008) .

In addition to the Rai and Binet scores (Rai et al 1975), many molecular and cytogenetic are used as prognostic factor in CLL, such as 17p, 11q and 13q deletions (Rosenquist et al., 2013 and Rossi et al., 2013), ZAP70
CD38 and IGVH (Lin et al., 2009, Chevallier et al., 2002 and Durig et al., 2003).

Despite the new targeted therapies and chemotherapeutical regimens approved in the management of CLL, the W\&W remains the standard treatment in asymptomatic and early stage CLL (Cheson et al , 1996) . This strategy is widely used to counteract the side effects of chemotherapy, since there is no difference in survival between this strategy and initiating chemotherapy at early and asymptomatic stages (International Workshop on Chronic Lymphocytic Leukemia, 1989).

In this paper, we are reporting for the first time the epidemiological, clinical, biological and therapeutic characteristics of patients with CLL in Lebanon and, consequently, the Middle East region. Next to evaluating 
those characteristics, the main objective of this study is to show the relevance of the W\&W strategy in the management of CLL in a developing country.

\section{Materials and Methods}

195 patients with CLL diagnosed in four medical institutions in Lebanon, between 1992 and 2013, were found to be eligible in this study after a computerized and manual search of the patients having CLL. The data was collected from the private clinic of the physicians and the archives of the hospitals using a pre-established Excel $^{\circledR}$ template.

The evaluated variables in this study were age of the patients diagnosed with CLL, their gender, RAI and Binet scores at diagnosis, $\mathrm{CBC}$ values, presence of hepatomegaly or splenomegaly, performance of bone marrow biopsy or peripheral blood flux cytometry for diagnosis, adoption of W\&W strategy, different chemotherapeutical regimens used at diagnosis and after progression and the indications for treatment. The collected data was analysed using the SPSS 20 software.

\section{Results}

Out of 95 patients diagnosed with CLL, 38 patients $(40 \%)$ were women and $57(60 \%)$ were men with a sex ratio of 1.5. The mean age of this population was 65.07 years with a minimum of 36 years and a maximum of 89 years. Less than $10 \%$ of patients were aged less than 50 years.

The mean lymphocyte count at diagnosis was 39885/ $\mathrm{mm}^{3}$ [1596-290000], the mean hemoglobin level was 12.69 $\mathrm{g} / \mathrm{dl}$ [6.2-17] and the mean platelet count was 191255/ $\mathrm{mm}^{3}$ [14000-458000]. 26.3\% of patients with CLL had splenomegaly, while only $7.4 \%$ had hepatomegaly. $33.7 \%$ had undergone a bone marrow biopsy or bone marrow aspiration, $66.3 \%$ a flow cytometry immunophenotyping on circulating blood and $5.3 \%$ a lymph node biopsy. $50.5 \%, 17.2 \%, 14 \%, 7.5 \%$ and $10.8 \%$ had a RAI score of $0,1,2,3,4$ respectively at diagnosis. $65.6 \%, 17.2 \%$ and $17.2 \%$ had a Binet score of I, II and III respectively (Table 1).

The W\&W Strategy was adopted in $62.4 \%$ of patients with a mean duration of 37.68 months [3-216]. The W\&W adoption was decreasing according to RAI score, as it was used in $82.6 \%, 73.33 \%, 46.15 \%, 14.28 \%$ and $0 \%$ of patients having a RAI score of $0,1,2,3$ and 4 respectively, with a statistically significant difference $(\mathrm{p}<0.05)$. Concerning Binet score, the $\mathrm{W} \& \mathrm{~W}$ was also decreasing as it was used in $80 \%, 46.66 \%$ and $6.25 \%$ of patients with grade I, II and III respectively; the difference was also statistically significant $(\mathrm{p}<0.05)$.

In 58 patients out of $95, \mathrm{~W} \& \mathrm{~W}$ strategy was adopted at diagnosis; 33 patients received chemotherapy directly after the diagnosis. 17 patients out of $58(29.3 \%)$ received chemotherapy after W\&W. So, 50 patients out $95(52.6 \%)$ received chemotherapy, at diagnosis or after $\mathrm{W} \& \mathrm{~W}$.

The indications for treatment by chemotherapeutical regimens in these patients were as follows: associated anemia and thrombocytopenia (32.7\%), isolated anemia
Table 1. Demographic, Biological and Clinical Characteristics of CLL Patients

\begin{tabular}{ll}
\hline Age & 65.07 years [36-89] \\
Gender & Female $40 \%$ (38 patients) \\
& Male $60 \%(57$ patients $)$ \\
Lymphocytes Count & $39885 / \mathrm{mm}^{3}[1596-290000]$ \\
Hemoglobin level & $12.69 \mathrm{~g} / \mathrm{dl}[6.2-17]$ \\
Platelet Count & $191255 / \mathrm{mm}^{3}[14000-458000]$ \\
Splenomegaly & $26.3 \%(25$ patients $)$ \\
\hline
\end{tabular}

Table 2. Indications for Treatment by Chemotherapeutical Regimens

\begin{tabular}{lc}
\hline Indications for treatment & Incidence \\
\hline Anemia and thrombocytopenia & $32.70 \%$ \\
Anemia & $21.10 \%$ \\
Presence of lymph nodes & $18.40 \%$ \\
Lymphocytosis & $13.20 \%$ \\
Thrombocytopenia & $10.50 \%$ \\
Presence of blasts & $2(5.3 \%)$ \\
Fatigue & $2(5.3 \%)$ \\
Other causes & $1(2.6 \%)$ \\
\hline
\end{tabular}

Table 3. Chemotherapeutical Regimens Used for the Treatment of CLL

\begin{tabular}{lc}
\hline Chemotherapeutical regimens & Incidence \\
\hline Chlorambucil & $26.50 \%$ \\
Flufarabine-Cyclophosphamide-Rituximab (FCR) & $16.30 \%$ \\
CHOP ou R-CHOP & $16.30 \%$ \\
Cyclophosphamide & $16.30 \%$ \\
Cyclophosphamide-Vincristine & $8.20 \%$ \\
FC (Flufarabine-Cyclophosphamide) & $8.20 \%$ \\
Other regimens & $8.20 \%$ \\
\hline
\end{tabular}

(21.1\%), presence of disturbing lymph nodes (18.4\%), lymphocytosis $(13.2 \%)$ isolated thrombocytopenia $(10.5 \%)$, presence of blasts $(5.3 \%)$ and fatigue $(5.3 \%)$ (Table 2). The most frequent chemotherapies used in the treatment of CLL were Chlorambucil in first position, Flufarabine-Cyclophosphamide-Rituximab (FCR), CHOP or R-CHOP and Cyclophosphamide in the second position and Flufarabine-Cyclophosphamide in the third position. Note that Chlorambucil was used in patients treated before the year 2000 and Flufarabine-CyclophosphamideRituximab in patients after 2000 (Table 3).

The median overall survival of patients diagnosed with CLL is 33 months with a minimum of 2 months and a maximum of 216 months. The median duration postchemotherapy regimen is also 33 months with a minimum of 0 and a maximum of 192 months.

\section{Discussion}

When comparing the CLL patients' epidemiological, clinical, biological and therapeutic results at diagnosis in our study to other publications, many specific characteristics related to our CLL patients are highlighted.

The sex ratio of CLL patients in our study is 1.5 , lower when compared to the literature, where the reported sex ratio is between 1.7 and 2 . The mean age of the studied 
population is 65 years, which is lower than the mean age reported in developed countries. The lower mean age at diagnosis can be attributed to a globally younger Lebanese population. However, when comparing to the Iranian and Turkish population, the mean age of diagnosis of CLL of these populations is similar to the Lebanese population due to a similar age distribution in these populations (Pamuk et al., 2004 and Kermani et al., 2007).

Concerning diagnostic modalities we can note an excessive use of bone marrow biopsy and aspiration compared to a moderate use of flow cytometry immunophenotyping at diagnosis, knowing that the use of bone marrow biopsy or aspiration is not indicated during a CLL work-up, while the flow cytometry immunophenotyping is necessary to confirm the diagnosis of CLL (Hallek et al., 2013).

In the literature, the Rai score at diagnosis for CLL patients was historically reported as follows: $25 \%$ of cases were diagnosed at early stages, $50 \%$ at intermediate stages and 25\% at advanced stages (Rai et al 1975). The results in our study were different as more than $50 \%$ of the patients were diagnosed at an early stage, $31 \%$ at an intermediate stage and $18 \%$ at an advanced stage. Those results can be explained by the Lebanese liberal medical care system and the medicophilic character of Lebanese population, both leading to more frequent blood tests.

The percentage of $\mathrm{W} \& \mathrm{~W}$ adoption varies between different populations. In the US, this strategy is adopted in more than $90 \%$ of CLL patients (Hess et al., 2010), whereas in Turkey it is used in 52\% (Pamuk et al., 2004). In Lebanon, W\&W was adopted in $62 \%$ of the CLL population. These differences can be explained by the better follow-up and screening of patients in developed countries compared to Middle Eastern countries.

Due to the long interval of including patients to this study from 1992 to 2013, many different chemotherapeutical regimens administered to the patients diagnosed with CLL were noted. Before the era of FlufarabineCyclophosphamide-Rituximab, the Chlorambucil was the most frequently used chemotherapy in first line. After the approval of Flufarabine-Cyclophosphamide-Rituximab as a standard of care, the majority of the patients beneficiated from this regimen.

The major limitation of our study is the long interval of recruitment from 1992 to 2013, where different chemotherapeutical regimens were used. Another limitation is the absence of molecular and cytogenetic factors in the studied population, because of the unavailability of these tests at the diagnosis of the majority of these patients.

This retrospective review of CLL clinical practice approach showed an important implementation of the $\mathrm{W} \& \mathrm{~W}$ strategy with a long duration especially in early stages with low RAI or Binet score.

\section{References}

Binet JL, Auquier A, Dighiero G, et al (1981) A new prognostic classification of chronic lymphocytic leukemia derived from a multivariate survival analysis. Cancer, $\mathbf{4 8}, 198$.

Chevallier P, Penther D, Avet-Loiseau H, et al (2002). CD38 expression and secondary $17 \mathrm{p}$ deletion are important prognostic factors in chronic lymphocytic leukaemia. $\mathrm{Br} J$ Haematol, 116, 142.

Cheson BD, Bennett JM, Grever M, et al (1996). National Cancer Institute-sponsored Working Group guidelines for chronic lymphocytic leukemia: revised guidelines for diagnosis and treatment. Blood, 87, 4990.

Chronic lymphocytic leukemia: recommendations for diagnosis, staging, and response criteria (1989). International Workshop on Chronic Lymphocytic Leukemia. Ann Intern Med, 110, 236.

Durig J, Nuckel H, Cremer M, et al (2003). ZAP-70 expression is a prognostic factor in chronic lymphocytic leukemia. Leukemia, 17, 2426.

Hallek M, Cheson BD, Catovsky D, et al (2008). Guidelines for the diagnosis and treatment of chronic lymphocytic leukemia: a report from the international workshop on chronic lymphocytic leukemia updating the national cancer institute-working group 1996 guidelines. Blood, 111, 5446.

Hernández JA, Land KJ, McKenna RW (1995). Leukemias, myeloma, and other lymphoreticular neoplasms. Cancer, 75, 381 .

Hess G, Chen C, Satram-Hoang S, Reyes C (2010). Characteristics and treatment patterns in patients newly diagnosed with chronic lymphocytic leukemia (CLL). Abstract e16561 in ASCO.

Kermani IA, Dehdilani M, Dolatkhah R (2007). Chronic lymphocytic leukemia in the recent 10 years and treatment effects of Fludarabin. Asian Pac J Cancer Prev, 8, 367-71.

Lin KI, Tam CS, Keating MJ, et al (2009). Relevance of the immunoglobulin VH somatic mutation status in patients with chronic lymphocytic leukemia treated with fludarabine, cyclophosphamide, and rituximab or related chemoimmunotherapy regimens. Blood, 113, 3168 .

Mhaskar AR, Quinn G, Vadaparampil S, et al (2010). Timing of first-line cancer treatments - early versus late - a systematic review of phase III randomized trials. Cancer Treat Rev, 36, 621-8.

Pamuk ON, Pamuk GE, Soysal T, et al (2004). Chronic lymphocytic leukemia in Turkey: experience of a single center in Istanbul. South Med J, 97, 240-5.

Rai KR, Sawitsky A, Cronkite EP, et al (1975). Clinical staging of chronic lymphocytic leukemia. Blood, 46, 219.

Siegel RL, Miller KD, Jemal A (2015). Cancer statistics, 2015. CA Cancer J Clin, $\mathbf{6 5}, 5$.

Rosenquist R, Cortese D, Bhoi S, et al (2013). Prognostic markers and their clinical applicability in chronic lymphocytic leukemia: where do we stand? Leuk Lymphoma, 54, 2351-64.

Rossi D, Rasi S, Spina V, et al (2013). Integrated mutational and cytogenetic analysis identifies new prognostic subgroups in chronic lymphocytic leukemia. Blood, 121, 1403.

Smith A, Howell D, Patmore R, et al (2011). Incidence of haematological malignancy by sub-type: a report from the Haematological Malignancy Research Network. $\mathrm{Br} J$ Cancer, 105, 1684. 